\title{
ON EXPLICIT AND NUMERICAL SOLVABILITY OF PARABOLIC INITIAL-BOUNDARY VALUE PROBLEMS
}

\author{
ALEXANDER KOZHEVNIKOV AND OLGA LEPSKY
}

Received 26 July 2005; Revised 15 January 2006; Accepted 22 March 2006

A homogeneous boundary condition is constructed for the parabolic equation $\left(\partial_{t}+I-\right.$ $\Delta) u=f$ in an arbitrary cylindrical domain $\Omega \times \mathbb{R}\left(\Omega \subset \mathbb{R}^{n}\right.$ being a bounded domain, $I$ and $\Delta$ being the identity operator and the Laplacian) which generates an initial-boundary value problem with an explicit formula of the solution $u$. In the paper, the result is obtained not just for the operator $\partial_{t}+I-\Delta$, but also for an arbitrary parabolic differential operator $\partial_{t}+A$, where $A$ is an elliptic operator in $\mathbb{R}^{n}$ of an even order with constant coefficients. As an application, the usual Cauchy-Dirichlet boundary value problem for the homogeneous equation $\left(\partial_{t}+I-\Delta\right) u=0$ in $\Omega \times \mathbb{R}$ is reduced to an integral equation in a thin lateral boundary layer. An approximate solution to the integral equation generates a rather simple numerical algorithm called boundary layer element method which solves the 3D Cauchy-Dirichlet problem (with three spatial variables).

Copyright (C 2006 A. Kozhevnikov and O. Lepsky. This is an open access article distributed under the Creative Commons Attribution License, which permits unrestricted use, distribution, and reproduction in any medium, provided the original work is properly cited.

\section{Introduction}

It is well known that the initial-boundary value problem with the Dirichlet/Neumann boundary condition for the parabolic equation $\left(\partial_{t}+I-\Delta\right) u=f$ can be solved using the Green function. But the Green function can be found explicitly just for a few very specific domains $\Omega$ such as balls and half-spaces. Unfortunately, in the case of an arbitrary domain $\Omega$, there is no explicit formula for the solution.

In this paper, the following question is investigated. How can one define boundary conditions for an arbitrary domain $\Omega$ in order to obtain an explicitly solvable initial boundary value problem? An answer is obtained not just for the operator $\partial_{t}+I-\Delta$, but also for a rather general parabolic differential operator of the form $\partial_{t}+A$, where $A$ is an elliptic differential operator of even order with constant coefficients. Similar questions for elliptic boundary value problems have been investigated in [4]. 
It turns out - and this is the first result of the paper - that by replacing the DirichletNeumann boundary condition with a more complicated homogeneous equation on boundary, we obtain an explicitly solvable initial-boundary value problem. Moreover, the solution can be represented by an explicit formula similar to the solution of the equation $\left(\partial_{t}+I-\Delta\right) u=f$ over the whole space $\mathbb{R}^{n+1}$. The anisotropic Sobolev spaces are very natural for solvability of parabolic initial-boundary value problems. These spaces as well as the solvability have been investigated by Slobodeckiı̌ [9], Agranovič and Višik [1], V. A. Solonnikov (see, e.g., [5]), Lions and Magenes [6], Grubb [3], and Eidelman and Zhitarashu [2].

Let $\mathbf{n}$ be the unit normal to $\partial \Omega$, pointing towards the exterior of $\Omega$. To state the boundary condition which gives an explicit solution, let us denote by $\Psi_{-}$the DirichletNeumann operator which maps the boundary trace $\left.v_{-}\right|_{\partial \Omega \times \mathbb{R}_{+}}$of a function $v_{-}$satisfying the equation $\left(\partial_{t}+I-\Delta\right) v_{-}(x, t)=0\left((x, t) \in \Omega_{-}:=\complement \bar{\Omega}\right)$ into the boundary trace of its normal derivative $\left.\partial_{\mathbf{n}} \nu_{-}\right|_{\partial \Omega \times \mathbb{R}_{+}}$. More precisely, $\Psi_{-}$is a composition of a Poisson operator $[3$, Section 3] solving the problem

$$
\begin{gathered}
\left(\partial_{t}+I-\Delta\right) v_{-}(x, t)=0, \quad(x, t) \in \Omega_{-} \times \mathbb{R}_{+}, \\
v_{-}(x, 0)=0, \quad x \in \Omega_{-}, \\
v_{-}(x, t)=g(x, t), \quad x \in \partial \Omega
\end{gathered}
$$

and the trace operator $\left.\partial_{\mathbf{n}} v_{-}\right|_{\partial \Omega \times \mathbb{R}_{+}}$. Replacing $\Omega_{-}$in $(1.1)$ by $\Omega_{+}:=\Omega$, we obtain the operator $\Psi_{+}$. We show that the equation $\left(\partial_{t}+I-\Delta\right) u_{ \pm}(x, t)=f_{ \pm}(x, t)\left((x, t) \in \Omega_{ \pm} \times \mathbb{R}_{+}\right)$ under the homogeneous boundary condition $\partial_{\mathbf{n}} u_{ \pm}-\Psi_{\mp} u_{ \pm}=0$ on $\partial \Omega \times \mathbb{R}_{+}$has a unique solution belonging to an anisotropic Sobolev space. We note that the latter boundary condition is parabolic, that is, the corresponding Lopatinskii condition holds.

As a consequence-and this is the second of two main results of the paper-the interior/exterior Cauchy-Dirichlet problem

$$
\begin{gathered}
\left(\partial_{t}+I-\Delta\right) v_{ \pm}(x, t)=0, \quad(x, t) \in \Omega_{ \pm} \times \mathbb{R}_{+}, \\
v_{ \pm}(x, 0)=0, \quad x \in \Omega_{ \pm}, \\
v_{ \pm}(x, t)=g(x, t), \quad x \in \partial \Omega,
\end{gathered}
$$

is reduced to an integral equation in a thin exterior/interior lateral boundary layer of $\partial \Omega \times \mathbb{R}_{+}$. An approximate solution of the integral equation generates a rather simple numerical algorithm solving the interior/exterior Cauchy-Dirichlet problem in the case of $\Omega_{ \pm} \subset \mathbb{R}^{n}(n=2,3)$. The algorithm is different from the methods of finite differences, finite elements, boundary elements, or difference potentials. It can be called boundary layer element method. It is shown that any solution of the interior/exterior Cauchy-Dirichlet problem with zero initial data is represented in the form of the layer potential with an unknown density supported in an arbitrarily thin exterior/interior boundary layer. Such 
layer potential representation is simpler than the representation by either simple-layer or double-layer potentials in the boundary element method or related representation by difference potentials [8]. Further, the standard cubic grid, just as in finite difference method, is used for the calculation of the unknown density. We reduce the problem to a linear system of $N M$ equations, where $N$ is the number of cubic cells inside the thin exterior boundary layer to $\Omega \subset \mathbb{R}^{3}$, and $M$ is the number of time levels. It turns out that the system has a lower block-triangular matrix with $M$ equal diagonal blocks. Each block is a square matrix of order $N$. The solution of the system needs const $\cdot N^{3} M$ operations and is obtained using a standard PC for $N=536, M=10$. Examples of an accuracy of the method are presented.

The first equation $\left(\partial_{t}+I-\Delta\right) v_{ \pm}(x, t)=0$ in (1.2) may be replaced by the usual heat equation $\left(\partial_{t}-\Delta\right) u_{ \pm}(x, t)=0$ due to the fact that if $v(x, t)$ satisfies (1.2), then the function $u_{ \pm}=e^{-t} v_{ \pm}$is a solution to the following Cauchy-Dirichlet problem:

$$
\begin{gathered}
\left(\partial_{t}-\Delta\right) u_{ \pm}(x, t)=0, \quad(x, t) \in \Omega_{ \pm} \times \mathbb{R}_{+}, \\
u_{ \pm}(x, 0)=0, \quad x \in \Omega_{ \pm}, \\
u_{ \pm}(x, t)=e^{t} g(x, t), \quad x \in \partial \Omega .
\end{gathered}
$$

In the next section, instead of $I-\Delta$, we consider a more general case, that is, an arbitrary invertible elliptic differential operator $A$ of even order $2 m$ with constant coefficients. We prove that under appropriate homogeneous boundary conditions, the operator $\partial / \partial t+A$ generates an isomorphism between anisotropic Sobolev spaces with an explicit formula for the inverse operator.

In [4], similar results are obtained for the elliptic equation $(I-\Delta) u=f$, as well as for its generalization $(I-A) u=f$.

\section{Theorem on explicit solvability}

Some prerequisites, such as the definition of the weighted anisotropic Sobolev spaces as well as a result on solvability of the Cauchy-Dirichlet problem, are collected below before the statement of Theorem 2.2.

Let $A$ be a linear differential operator in $\mathbb{R}^{n}$ of an even order $2 m\left(m \in \mathbb{N}_{+}:=\{1,2, \ldots\}\right)$, with constant coefficients $a_{\alpha} \in \mathbb{C}$, that is, $A:=A(D):=\sum_{|\alpha| \leq 2 m} a_{\alpha} D^{\alpha}$, where $\alpha$ is a multiindex, that is, $\alpha:=\left(\alpha_{1}, \ldots, \alpha_{n}\right), \alpha_{j} \in \mathbb{N}:=\{0,1,2, \ldots\},|\alpha|:=\alpha_{1}+\cdots+\alpha_{n}, i:=\sqrt{-1} ; D_{j}:=$ $i^{-1} \partial / \partial x_{j} ; D^{\alpha}:=D_{1}^{\alpha_{1}} D_{2}^{\alpha_{2}} \cdots D_{n}^{\alpha_{n}}$.

Let $\partial \Omega$ be a closed compact infinitely smooth surface in $\mathbb{R}^{n}$ bounding a domain $\Omega_{+}$ and let $\Omega_{-}$be the complement of $\bar{\Omega}_{+}$in $\mathbb{R}^{n}$, where $\bar{\Omega}_{+}$is the closure of $\Omega_{+}$.

We consider two equations:

$$
\left(\partial_{t}+A(D)\right) u_{ \pm}(x, t)=f_{ \pm}(x, t), \quad \text { either }(x, t) \in \Omega_{+} \times \mathbb{R}_{+} \text {or }(x, t) \in \Omega_{-} \times \mathbb{R}_{+},
$$

in short $\left(\partial_{t}+A(D)\right) u_{ \pm}(x, t)=f_{ \pm}(x, t), x \in \Omega_{ \pm} \times \mathbb{R}_{+}$.

The polynomials $a(\xi):=\sum_{|\alpha| \leq 2 m} a_{\alpha} \xi^{\alpha}$ and $a_{0}(\xi):=\sum_{|\alpha|=2 m} a_{\alpha} \xi^{\alpha}$ are called, respectively, the symbol and the principal symbol of $A$. Let the following condition be satisfied. 
4 On explicit and numerical solvability

Condition 2.1. There is a constant $c>0$ such that

$$
\left|q^{2 m}+a_{0}(\xi)\right| \geq c(|\xi|+|q|)^{2 m} \text { for } \xi \in \mathbb{R}^{n},|\arg q| \leq \frac{\pi}{4 m} .
$$

Weighted anisotropic Sobolev spaces. Let $0 \leq s \in \mathbb{R}$ and $\Omega$ coincide with either $\mathbb{R}^{n}$ or $\mathbb{R}_{+}^{n}:=$ $\left\{x=\left(x_{1}, \ldots, x_{n}\right) \in \mathbb{R}^{n}: x_{n}>0\right\}$ or $\Omega_{ \pm}$. Let $H^{s}(\Omega)$ denote the usual Sobolev spaces over $\Omega$. Following $[1,9]$, we consider the anisotropic Sobolev space $H^{(s, s / d)}(\Omega \times \mathbb{R})(0 \leq s \in$ $\left.\mathbb{R}, d \in \mathbb{N}_{+}\right)$, that is, the completion of the set of all smooth functions $u(x, t)$ with respect to the norm

$$
\|u\|_{H^{(s, s / d)}}^{2}(\Omega \times \mathbb{R}):=\int_{0}^{\infty}\|u(x, t)\|_{H^{s}(\Omega)}^{2} d t+\int_{\Omega}\|u(x, t)\|_{H^{s / d}(\mathbb{R})}^{2} d x .
$$

Let $H^{s}(\partial \Omega)$ denote the Sobolev spaces over the smooth surface $\partial \Omega$, and let $H^{(s, s / d)}(\partial \Omega$ $\times \mathbb{R})$ be the anisotropic Sobolev space of functions $u\left(x^{\prime}, t\right)\left(x^{\prime} \in \partial \Omega\right)$ with the norm

$$
\|u\|_{H^{(s, s / d)}(\partial \Omega \times \mathbb{R})}^{2}:=\int_{0}^{\infty}\left\|u\left(x^{\prime}, t\right)\right\|_{H^{s}(\partial \Omega)}^{2} d t+\sum_{j} \int_{\partial \Omega}\left\|\varphi_{j}\left(x^{\prime}\right) u\left(x^{\prime}, t\right)\right\|_{H^{s / d}(\mathbb{R})}^{2} d x^{\prime},
$$

where $\left\{\varphi_{j}\right\}$ is a partition of unity on $\partial \Omega$ such that

$$
\|u\|_{H^{s}(\partial \Omega)}^{2}:=\sum_{j}\left\|\varphi_{j}\left(x^{\prime}\right) u\right\|_{H^{s}\left(\mathbb{R}^{n-1}\right)}^{2}
$$

For $\delta \geq 0$, let $H_{(0)}^{(s, s / d)}\left(\Omega \times \mathbb{R}_{+}, \delta\right)\left(0 \leq s \in \mathbb{R}, d \in \mathbb{N}_{+}\right)$denote the weighted anisotropic Sobolev space of all functions $u(x, t)$ defined in $\Omega \times \mathbb{R}$ and equal zero for $t<0$ and such that $e^{-\delta t} u \in H^{(s, s / d)}(\Omega \times \mathbb{R})$. The norm in this space is defined by

$$
\|u\|_{H_{(0)}^{(s, s)}\left(\Omega \times \mathbb{R}_{+}, \delta\right)}:=\left\|e^{-\delta t} u\right\|_{H^{(s, s / d)}(\Omega \times \mathbb{R})} .
$$

Similarly, $H_{(0)}^{(s, s / d)}\left(\partial \Omega \times \mathbb{R}_{+}, \delta\right)\left(0 \leq s \in \mathbb{R}, d \in \mathbb{N}_{+}\right)$denotes the weighted anisotropic Sobolev space of all functions $u\left(x^{\prime}, t\right)$ defined in $\partial \Omega \times \mathbb{R}$ and equal zero for $t<0$ and such that $e^{-\delta t} u \in H^{(s, s / d)}(\partial \Omega \times \mathbb{R})$. The norm in this space is defined by

$$
\|u\|_{H_{(0)}^{(s, s)}\left(\partial \Omega \times \mathbb{R}_{+}, \delta\right)}:=\left\|e^{-\delta t} u\right\|_{H^{(s, s / d)}(\partial \Omega \times \mathbb{R})} .
$$

Let $D_{\mathbf{n}}:=i^{-1} \partial_{\mathbf{n}}$. It is known that the trace operators are continuos and surjective:

$$
\left.u \longmapsto D_{\mathbf{n}}^{j} u\right|_{\partial \Omega \times \mathbb{R}_{+}}: H_{(0)}^{(s, s / d)}\left(\Omega_{ \pm} \times \mathbb{R}_{+}, \delta\right) \longrightarrow H_{(0)}^{(s-j-1 / 2,(s-j-1 / 2) / d)}\left(\partial \Omega \times \mathbb{R}_{+}, \delta\right)
$$

for $s>j-1 / 2$.

Let

$$
g_{j}\left(x^{\prime}, t\right) \in H_{(0)}^{(2 m-j-1 / 2,(2 m-j-1 / 2) /(2 m))}\left(\partial \Omega \times \mathbb{R}_{+}, \delta\right) \quad(j=1, \ldots, m-1) .
$$


For the homogeneous equations (2.1), we consider two Cauchy-Dirichlet problems (one over $\Omega_{+} \times \mathbb{R}_{+}$and the other over $\Omega_{-} \times \mathbb{R}_{+}$):

$$
\begin{gathered}
\quad\left(\partial_{t}+A(D)\right) v_{ \pm}=0, \quad \text { in } \Omega_{ \pm} \times \mathbb{R}_{+}, \\
D_{\mathbf{n}}^{j} v_{ \pm}=g_{j} \quad(j=0,1, \ldots, m-1), \quad \text { on } \partial \Omega \times \mathbb{R}_{+}, \\
\left.v_{ \pm}\right|_{t=0}=0, \quad \text { on } \Omega_{ \pm} .
\end{gathered}
$$

By the Laplace transform, the Cauchy-Dirichlet problem (2.10) can be formally reduced to the corresponding boundary value problem depending on the complex parameter $p$ :

$$
\begin{gathered}
(p+A(D)) V_{ \pm}(x, p)=0, \quad x \in \Omega_{ \pm}, \\
D_{\mathbf{n}}^{j} V_{ \pm}\left(x^{\prime}, p\right)=G_{j}\left(x^{\prime}, p\right) \quad(j=0,1, \ldots, m-1), x^{\prime} \in \partial \Omega .
\end{gathered}
$$

Under condition (2.2), there exists $\gamma>0$ such that for $\operatorname{Re} p>\gamma$, the problem (2.11) has a unique solution belonging to an appropriate Sobolev space. This assertion has been stated in [10, Theorem 1.4], where it is noted that for the bounded domain $\Omega_{+}$, this fact is proved in [1], however, the localization technique used in [1] enables one to establish the assertion for the unbounded domain $\Omega_{-}$. In view of this and using [1, Theorem 9.2], we obtain that the Cauchy-Dirichlet problem $(2.10)$ has a unique solution $v_{ \pm} \in H_{(0)}^{(2 m, 1)}\left(\Omega_{ \pm} \times\right.$ $\left.\mathbb{R}_{+}, \delta\right)$.

By $\mathscr{D}$ and $\mathcal{N}$, we denote the vector operators

$$
\begin{aligned}
\mathscr{D} & :=\left.\left(I, D_{\mathbf{n}}, \ldots, D_{\mathbf{n}}^{m-1}\right)\right|_{\partial \Omega \times \mathbb{R}_{+}}, \\
\mathcal{N} & :=\left.\left(D_{\mathbf{n}}^{m}, \ldots, D_{\mathbf{n}}^{2 m-1}\right)\right|_{\partial \Omega \times \mathbb{R}_{+}} .
\end{aligned}
$$

It is known that if $v_{ \pm}(x, t)$ is the solution of (2.10), the vectors $\mathscr{D} v_{ \pm}$and $\mathcal{N} v_{ \pm}$are related by the equation

$$
\mathcal{N} v_{ \pm}=\Psi_{ \pm}\left(\mathscr{D} v_{ \pm}\right)
$$

Here $\Psi_{ \pm}:=\left\{\Psi_{ \pm, j k}\right\}_{j, k=1, \ldots, m}$ is an $m \times m$ matrix operator acting on $\partial \Omega \times \mathbb{R}_{+}$which can be called a Dirichlet-to-Neumann mapping. We note that $\Psi_{ \pm, j k}$ is an operator of order $\varkappa:=m+j-k$ mapping the space $H_{(0)}^{(s, s /(2 m))}\left(\Omega_{ \pm} \times \mathbb{R}_{+}\right)$to $H_{(0)}^{(s-\varkappa,(s-\varkappa) /(2 m))}\left(\Omega_{ \pm} \times \mathbb{R}_{+}\right)$.

Let $\Phi:=\left\{\Phi_{j k}\right\}_{j, k=1, \ldots, m}$ be another $m \times m$ matrix operator acting on $\partial \Omega \times \mathbb{R}_{+}$such that ord $\Phi_{j k}=m+j-k$.

For $f_{+}(x, t) \in L_{2}\left(\Omega_{+} \times \mathbb{R}_{+}, \delta\right)$, we consider the following conjugation problem (a particular case of a conjugation problem studied in [2]):

$$
\begin{gathered}
\left(\partial_{t}+A(D)\right) u_{+}(x, t)=f_{+}(x, t), \quad(x, t) \in \Omega_{+} \times \mathbb{R}, \\
\left(\partial_{t}+A(D)\right) u_{-}(x, t)=0, \quad(x, t) \in \Omega_{-} \times \mathbb{R}, \\
\mathscr{D} u_{+}\left(x^{\prime}, t\right)-\mathscr{D} u_{-}\left(x^{\prime}, t\right)=0, \quad\left(x^{\prime}, t\right) \in \partial \Omega_{+} \times \mathbb{R}, \\
\mathcal{N} u_{+}\left(x^{\prime}, t\right)-\Phi\left(\mathscr{D} u_{+}\right)\left(x^{\prime}, t\right)=0, \quad\left(x^{\prime}, t\right) \in \partial \Omega_{+} \times \mathbb{R}, \\
u_{+}(x, 0)=0, \quad x \in \Omega_{+}, \\
u_{-}(x, 0)=0, \quad x \in \Omega_{-} .
\end{gathered}
$$


We will call the function $u(x, t)$ a solution to the conjugation problem if with some $\delta>0$,

$$
u(x, t):= \begin{cases}u_{+}(x, t) \in H_{(0)}^{(2 m, 1)}\left(\Omega_{+} \times \mathbb{R}_{+}, \delta\right), & (x, t) \in \Omega_{+} \times \mathbb{R}, \\ u_{-}(x, t) \in H_{(0)}^{(2 m, 1)}\left(\Omega_{-} \times \mathbb{R}_{+}, \delta\right), & (x, t) \in \Omega_{-} \times \mathbb{R},\end{cases}
$$

and (2.14)-(2.19) hold.

Theorem 2.2. Let Condition 2.1 be satisfied.

Then for any $f_{+}(x, t) \in L_{2}\left(\Omega_{+} \times \mathbb{R}_{+}, \delta\right)$ with some $\delta>0$, the conjugation problem (2.14)(2.19) has a "smooth" solution $u(x, t) \in H_{(0)}^{(2 m, 1)}\left(\mathbb{R}^{n} \times \mathbb{R}_{+}, \delta\right)$ if and only if $u(x, t)$ satisfies (2.17) with $\Phi$ replaced by $\Psi_{-}$.

If $\Phi=\Psi_{-}$, the solution $u(x, t)$, and consequently $u_{+}(x, t)$ and $u_{-}(x, t)$, can be represented as

$$
u(x, t)=\int_{0}^{t} d \tau \int_{\mathbb{R}^{n}} \Xi(t-\tau, x-\xi) f_{+}(\tau, \xi) d \xi
$$

where the function $\Xi(t, x)$ is the fundamental solution to the equation $\left(\partial_{t}+A(D)\right) u=f_{+}$, that is,

$$
\Xi(x, t)=\mathscr{F}_{(\xi, \tau) \rightarrow(x, t)}^{-1} \frac{1}{i \tau+a(\xi)},
$$

and $\mathscr{F}_{(\xi, \tau) \rightarrow(x, t)}^{-1}$ denotes the inverse Fourier transform.

If $\Phi=\Psi_{-}$, the Cauchy-Dirichlet problem (2.14), (2.17), and (2.18) is parabolic (i.e., the corresponding parameter-dependent problem satisfies the Lopatinskii condition) and uniquely solvable and the operator $\mathscr{D} u_{+}$maps surjectively the set of all solutions $u_{+}(x, t) \in H_{(0)}^{(2 m, 1)}$ $\left(\Omega_{+} \times \mathbb{R}_{+}, \delta\right)$ satisfying $(2.17)$ onto the space

$$
\prod_{j=0}^{m-1} H_{(0)}^{(2 m-j-1 / 2,(2 m-j-1 / 2) / 2 m)}\left(\partial \Omega \times \mathbb{R}_{+}, \delta\right)
$$

Proof. Let $u(x, t) \in H_{(0)}^{(2 m, 1)}\left(\mathbb{R}^{n} \times \mathbb{R}_{+}, \delta\right)$ be a solution to the conjugation problem (2.14)(2.19). Then, by (2.15) and (2.16), we get

$$
\Psi_{-}\left(\mathscr{D} u_{+}\right)\left(x^{\prime}, t\right)=\Psi_{-}\left(\mathscr{D} u_{-}\right)\left(x^{\prime}, t\right)=\mathcal{N} u_{-}\left(x^{\prime}, t\right)
$$

Since $u(x, t) \in H^{(2 m, 1)}\left(\mathbb{R}^{n} \times \mathbb{R}_{+}, \delta\right), \mathcal{N} u_{-}\left(x^{\prime}, t\right)=\mathcal{N} u_{+}\left(x^{\prime}, t\right)$. By $(2.17)$, we have

$$
\Psi_{-}\left(\mathscr{D} u_{+}\right)\left(x^{\prime}, t\right)=\mathcal{N} u_{-}\left(x^{\prime}, t\right)=\mathcal{N} u_{+}\left(x^{\prime}, t\right)=\Phi\left(\mathscr{D} u_{+}\right)\left(x^{\prime}, t\right)
$$

Therefore, $\Phi\left(\mathscr{D} u_{+}\right)\left(x^{\prime}, t\right)=\Psi_{-}\left(\mathscr{D} u_{+}\right)\left(x^{\prime}, t\right)$, which proves the necessity.

Now, let $u_{+}(x, t) \in H_{(0)}^{(2 m, 1)}\left(\Omega_{+} \times \mathbb{R}_{+}, \delta\right)$ and $u_{-}(x, t) \in H_{(0)}^{(2 m, 1)}\left(\Omega_{-} \times \mathbb{R}_{+}, \delta\right)$ satisfy (2.14)-(2.19) with $\Phi=\Psi_{-}$. In particular, $u_{+}(x, t)$ satisfies (2.14), (2.17), and (2.18), that 
is, $u_{+}(x, t)$ is a solution to the initial boundary value problem

$$
\begin{gathered}
\left(\partial_{t}+A(D)\right) u_{+}(x, t)=f_{+}(x, t), \quad(x, t) \in \Omega_{+} \times \mathbb{R}_{+}, \\
\mathcal{N} u_{+}(x, t)-\Psi_{-}\left(\mathscr{D} u_{+}(x, t)\right)=0, \quad(x, t) \in \partial \Omega_{+} \times \mathbb{R}_{+}, \\
u_{+}(x, 0)=0, \quad x \in \Omega_{+} .
\end{gathered}
$$

Following [1], to check the Lopatinskii condition, we suppose that $\Omega_{+}$is a hyperplane $x_{n}>0$ and replace $\partial_{t}$ by $q^{2 m}$, replace $A(D)=A\left(D^{\prime}, D_{n}\right)$ by its principal part $A_{0}\left(D^{\prime}, D_{n}\right)$, and then by $a_{0}\left(\xi^{\prime}, D_{n}\right)$. In this case, it is enough to check that for $f_{+}(x, t) \equiv 0$, the solution $u_{+}\left(x^{\prime}, x_{n}, q\right) \in H^{2 m}\left(\mathbb{R}^{n-1} \times \mathbb{R}_{+}\right)$to the problem

$$
\begin{gathered}
\left(q^{2 m}+A_{0}\left(D^{\prime}, D_{n}\right)\right) u_{+}\left(x^{\prime}, x_{n}, q\right)=0, \quad x^{\prime} \in \mathbb{R}^{n-1}, x_{n} \in \mathbb{R}_{+},|\arg q| \leq \frac{\pi}{4 m}, \\
D_{n}^{m+k} u_{+}\left(x^{\prime}, 0, q\right)-\Psi_{-}\left(D_{n}^{k} u_{+}\left(x^{\prime}, 0, q\right)\right)=0, \quad k=0,1, \ldots, m-1, \\
\lim _{x_{n} \rightarrow \infty} u_{+}\left(x^{\prime}, x_{n}, q\right)=0
\end{gathered}
$$

is the identical zero. Indeed, the Cauchy data of $u_{+}$belongs to the subspace

$$
\mathcal{N} u_{+}\left(x^{\prime}, q\right)-\Psi_{-}\left(\mathscr{D} u_{+}\right)\left(x^{\prime}, q\right)=0 \text {. }
$$

On the other hand, since $u_{+}$is the solution to the homogeneous equation

$$
\left(q^{2 m}+A_{0}(D)\right) u_{+}(x, q)=0
$$

its Cauchy's data belongs to the subspace

$$
\mathcal{N} u_{+}\left(x^{\prime}, q\right)-\Psi_{+}\left(\mathscr{D} u_{+}\right)\left(x^{\prime}, q\right)=0 \text {. }
$$

It is known [10, Proposition 2.3] that under Condition 2.1, the intersection of two subspaces consists solely of the zero element, that is, the Lopatinskii condition holds. This together means that the problem (2.26) is parabolic.

Now, let $f_{+}(x, t) \in L_{2}\left(\Omega_{+} \times \mathbb{R}_{+}, \delta\right)$ and $u_{+}(x, t) \in H_{(0)}^{(2 m, 1)}\left(\Omega_{+} \times \mathbb{R}_{+}, \delta\right)$ be a solution to the problem (2.26). By (2.8), we have

$$
\mathscr{D} u_{+} \in \prod_{j=0}^{m-1} H_{(0)}^{(2 m-j-1 / 2,(2 m-j-1 / 2) /(2 m))}\left(\partial \Omega \times \mathbb{R}_{+}, \delta\right)
$$

We denote by $u_{-}(x, t)$ the solution to the Cauchy-Dirichlet problem

$$
\begin{array}{cc}
\left(\partial_{t}+A(D)\right) u_{-}(x, t)=0, & (x, t) \in \Omega_{-} \times \mathbb{R}_{+}, \\
\mathscr{D} u_{-}\left(x^{\prime}, t\right)=\mathscr{D} u_{+}\left(x^{\prime}, t\right), & (x, t) \in \partial \Omega \times \mathbb{R}_{+}, \\
u_{-}(x, 0)=0, & x \in \Omega_{-} .
\end{array}
$$

There exists a unique solution to the latter problem $u_{-}(x, t) \in H_{(0)}^{(2 m, 1)}\left(\Omega_{-} \times \mathbb{R}_{+}, \delta\right)$. 
In view of the definition of the Dirichlet-to-Neumann mapping, we have

$$
\mathcal{N} u_{-}(x, t)=\Psi_{-}\left(\mathscr{D} u_{-}(x, t)\right)=\Psi_{-}\left(\mathscr{D} u_{+}(x, t)\right)=\mathcal{N} u_{+}(x, t)
$$

Therefore, we get for the functions $u_{ \pm}(x, t)$ belonging to $H_{(0)}^{(2 m, 1)}\left(\Omega_{ \pm} \times \mathbb{R}_{+}, \delta\right)$ that their normal derivatives up to order $2 m-1$ coincide on the interface $\partial \Omega$. From this follows that the function $u(x, t)$ defined by (2.20) belongs to $H_{(0)}^{(2 m, 1)}\left(\mathbb{R}^{n} \times \mathbb{R}_{+}, \delta\right)$. Moreover, $u(x, t)$ satisfies the equation

$$
\left(\partial_{t}+A(D)\right) u(x, t)=f(x, t), \quad(x, t) \in \mathbb{R}^{n} \times \mathbb{R},
$$

where

$$
f(x, t)= \begin{cases}f_{+}(x, t), & (x, t) \in \Omega_{+} \times \mathbb{R}_{+} \\ 0, & (x, t) \in \Omega_{-} \times \mathbb{R}_{+} .\end{cases}
$$

Thus, it was proved that each solution to (2.26) has a smooth extension which satisfies (2.34). Conversely, let us prove that each solution to (2.34) with $f_{+}(x, t) \in L_{2}\left(\Omega_{+} \times \mathbb{R}_{+}, \delta\right)$ satisfies (2.14)-(2.19). Indeed, since $u \in H_{(0)}^{(2 m, 1)}\left(\mathbb{R}^{n} \times \mathbb{R}_{+}, \delta\right)$, we have for $u_{+}=\left.u\right|_{\Omega_{+}}$and $u_{-}=\left.u\right|_{\Omega_{-}}$that

$$
\mathscr{D} u_{+}\left(x^{\prime}, t\right)=\mathscr{D} u_{-}\left(x^{\prime}, t\right), \quad \mathcal{N} u_{+}\left(x^{\prime}, t\right)=\mathcal{N} u_{-}\left(x^{\prime}, t\right)
$$

Since $\left(\partial_{t}+A(D)\right) u_{-}=0$, then $\mathcal{N} u_{-}\left(x^{\prime}, t\right)=\Psi_{-}\left(\mathscr{D} u_{-}\right)\left(x^{\prime}, t\right)$, and therefore $\mathcal{N} u_{+}\left(x^{\prime}, t\right)-$ $\Psi_{-}\left(\mathscr{D} u_{+}\right)\left(x^{\prime}, t\right)=0$. It follows that $u_{+}$and $u_{-}$satisfy (2.14)-(2.19). In particular, the restriction $u_{+}$of $u$ is a unique solution to (2.26). This means that the parabolic problem (2.26) has a unique solution $u_{+}(x, t)$ belonging to $H_{(0)}^{(2 m, 1)}\left(\Omega_{+} \times \mathbb{R}_{+}, \delta\right)$ for each right-hand side $f_{+}(x, t) \in L_{2}\left(\Omega_{+} \times \mathbb{R}_{+}, \delta\right)$. In view of $(2.34)$, we get the formula (2.21).

To prove the last sentence of the theorem, we use the known fact that the operator $\mathscr{D}$ maps surjectively the space $H_{(0)}^{(2 m, 1)}\left(\Omega_{+} \times \mathbb{R}_{+}, \delta\right)$ onto the space

$$
\prod_{j=0}^{m-1} H_{(0)}^{(2 m-j-1 / 2,(2 m-j-1 / 2) /(2 m))}\left(\partial \Omega \times \mathbb{R}_{+}, \delta\right) .
$$

Let us note that the boundary condition $\mathcal{N} u_{+}\left(x^{\prime}, t\right)=\Psi_{-}\left(\mathscr{D} u_{+}\right)\left(x^{\prime}, t\right)$ means that there are no constraints for the Dirichlet data $\mathscr{D} u_{+}\left(x^{\prime}, t\right)$ and just restrictions for the Neumann data $\mathcal{N} u_{+}\left(x^{\prime}, t\right)$. Therefore, the operator $\mathscr{D}$ maps surjectively the subspace of all solutions $u_{+}(x, t) \in H_{(0)}^{(2 m, 1)}\left(\Omega_{+} \times \mathbb{R}_{+}, \delta\right)$ satisfying $\mathcal{N} u_{+}\left(x^{\prime}, t\right)=\Psi_{-}\left(\mathscr{D} u_{+}\right)\left(x^{\prime}, t\right)$ onto the space (2.37).

\section{Numerical algorithm to the exterior/interior Cauchy-Dirichlet problem}

Near $\partial \Omega$ a normal vector field, $\mathbf{n}(x)=\left(n_{1}(x), \ldots, n_{n}(x)\right)$ is defined as follows: for $x_{0} \in \partial \Omega$, $\mathbf{n}\left(x_{0}\right)$ is the unit normal to $\partial \Omega$, pointing towards the exterior of $\Omega_{+}$. We set $\mathbf{n}(x):=\mathbf{n}\left(x_{0}\right)$ for $x$ of the form $x=x_{0}-s \mathbf{n}\left(x_{0}\right)=: \zeta\left(x_{0}, s\right)$, where $x_{0} \in \partial \Omega, s \in(-\varepsilon, \varepsilon)$. Here $\varepsilon>0$ is taken to be so small that the representation of $x$ in terms of $x_{0} \in \partial \Omega$ and $s \in(-\varepsilon, \varepsilon)$ is unique 
and smooth, that is, $\zeta$ is bijective and $C^{\infty}$ with $C^{\infty}$ inverse, from $\partial \Omega \times(-\varepsilon, \varepsilon)$ to the set $\zeta(\partial \Omega \times(-\varepsilon, \varepsilon)) \subset \mathbb{R}^{n}$. We denote by $\Omega_{+}^{\mathrm{bl}}:=\zeta(\partial \Omega \times(0, \varepsilon))$ the interior boundary layer to $\partial \Omega$, and denote by $\Omega_{-}^{\text {bl }}:=\zeta(\partial \Omega \times(-\varepsilon, 0))$ the exterior boundary layer to $\partial \Omega$. Let us note that $\Omega_{+}^{\mathrm{bl}} \subset \Omega_{+}$and $\Omega_{-}^{\mathrm{bl}} \subset \Omega_{-}$.

We denote the complements to the boundary layers by $\complement \bar{\Omega}_{ \pm}^{\mathrm{bl}}:=\mathbb{R}^{n} \backslash \bar{\Omega}_{ \pm}^{\mathrm{bl}}$, where $\bar{\Omega}_{ \pm}^{\mathrm{bl}}$ is the closure of $\Omega_{ \pm}^{\mathrm{bl}}$.

The theorem remains valid after the replacement of $\Omega_{+}$and $\Omega_{-}$, respectively, by the boundary layers $\Omega_{ \pm}^{\mathrm{bl}}$ and by $C \bar{\Omega}_{ \pm}^{\mathrm{bl}}$ in the statement of the theorem as well as in the statement of the conjugation problem (2.14)-(2.19). The proof is similar.

As a result, we obtain

$$
\begin{gathered}
u_{\mp}(x, t):=\int_{0}^{t} d \tau \int_{\Omega_{ \pm}^{\mathrm{bl}}} \Xi(t-\tau, x-\xi) f_{ \pm}(\tau, \xi) d \xi, \\
f_{ \pm} \in L_{2}\left(\Omega_{ \pm}^{\mathrm{bl}} \times \mathbb{R}_{+}, \delta\right) .
\end{gathered}
$$

Moreover, the function $u_{\mp}(x, t)$ is a solution to the Cauchy-Dirichlet problem

$$
\begin{gathered}
\left(\partial_{t}+A(D)\right) u_{\mp}(x, t)=0, \quad \text { in }(x, t) \in \Omega_{\mp} \times \mathbb{R}_{+}, u_{\mp} \in H^{(2 m, 1)}\left(\Omega_{\mp} \times \mathbb{R}_{+}, \delta\right), \\
\left.\left(D u_{\mp}\right)\right|_{\partial \Omega \times \mathbb{R}_{+}}=g, \\
\left.u_{\mp}\right|_{t=0}=0, \quad \text { on } \partial \Omega \times \mathbb{R}_{+},
\end{gathered}
$$

if and only if $f_{ \pm}(x, t)$ is a solution to the integral equation

$$
g(x, t)=\mathscr{D} \int_{0}^{t} d \tau \int_{\Omega_{ \pm}^{\mathrm{bl}}} \Xi(t-\tau, x-\xi) f_{ \pm}(\tau, \xi) d \xi \quad(x, t) \in \partial \Omega \times \mathbb{R}_{+} .
$$

Using the latter integral equation, let us construct an approximate solution to the Cauchy-Dirichlet problem

$$
\begin{gathered}
\left(\partial_{t}+I-\Delta\right) u_{+}(x, t)=0, \quad \text { in } \Omega_{+} \times(0, T), u_{+} \in H^{(2,1)}\left(\Omega_{+} \times(0, T)\right), \\
\left.u_{+}\right|_{\partial \Omega \times(0, T)}=g(x, t), \quad \text { on } \partial \Omega \times(0, T), g(x, t) \in H^{(3 / 2,3 / 4)}(\partial \Omega \times(0, T)), \\
\left.u_{+}\right|_{t=0}=0, \quad \text { on } \Omega_{+} .
\end{gathered}
$$

We consider a rectangular grid in the space $\mathbb{R}^{n}(n=1,2,3)$ and choose only those cells which belong to the thin exterior boundary layer $\Omega_{-}^{\mathrm{bl}}$. Denoting these cells by $\omega_{k}(k=$ $1, \ldots, N)$, we define the indicator $f_{k, r}(x, t)$ of the set $\omega_{k} \times\left(t_{r-1}, t_{r}\right)$, where $t_{j}=j T / M, j=$ $0,1, \ldots, M$, that is,

$$
f_{k, r}(x, t):= \begin{cases}1, & x \in \omega_{k} \times\left(t_{r-1}, t_{r}\right) \\ 0 & \text { otherwise }\end{cases}
$$

We introduce basic functions

$$
F_{k, r}(x, t):=\int_{0}^{t} d \tau \int_{\omega_{k}} \Xi(t-\tau, x-\xi) f_{k, r}(\tau, \xi) d \xi, \quad(x, t) \in \Omega_{+} \times \mathbb{R}_{+} .
$$


Let us note that since the functions $f_{k, r}$ are linearly independent and the operator in the right-hand side of (3.6) is invertible, then the functions $F_{k, r}$ are linearly independent.

It is not difficult to evaluate $F_{k, r}$ numerically. Indeed, the interior integral over the cell

$$
\omega_{k}=\left(\xi_{i-1}, \xi_{i}\right) \times\left(\eta_{i-1}, \eta_{i}\right) \times\left(\zeta_{i-1}, \zeta_{i}\right) \subset \mathbb{R}^{3}
$$

can be obtained as an algebraic expression including different values of the error function $\operatorname{erf}(x):=(2 / \sqrt{\pi}) \int_{0}^{x} \exp \left(-p^{2}\right) d p$. Let

$$
\Xi_{i}(\tau, \xi):=\operatorname{erf}\left(\frac{\xi_{i}-\xi}{2 \sqrt{\tau}}\right)-\operatorname{erf}\left(\frac{\xi_{i-1}-\xi}{2 \sqrt{\tau}}\right), \quad \xi, \xi_{i} \in \mathbb{R}, \tau>0 .
$$

Then for $x=(\xi, \eta, \zeta) \in \mathbb{R}^{3}$, we obtain, in view of (3.5),

$$
F_{k, r}(x, t)=F_{k, r}(\xi, \eta, \zeta, t)= \begin{cases}2^{-3} \int_{0}^{t-t_{m-1}} \Xi_{i}(\tau, \xi) \Xi_{j}(\tau, \eta) \Xi_{l}(\tau, \zeta) e^{\tau} d \tau, & t \in\left(t_{m-1}, t_{m}\right], \\ 2^{-3} \int_{t-t_{m}}^{t-t_{m-1}} \Xi_{i}(\tau, \xi) \Xi_{j}(\tau, \eta) \Xi_{l}(\tau, \zeta) e^{\tau} d \tau, & t>t_{m}, \\ 0, & t \leq t_{m-1} .\end{cases}
$$

The integral in the variable $\tau$ is calculated numerically. Analogously to the boundary element method, the basic functions $F_{k, r}(x, t)$ can be called lateral boundary layer elements. Looking for an approximate solution to (3.4) in the form

$$
v(x, t)=\sum_{k=1}^{N} \sum_{r=1}^{M} c_{k, r} F_{k, r}(x, t)
$$

we use $N M$ different points $\left(x_{j}, t_{i}\right) \in \partial \Omega \times(0, T)(j=1, \ldots, N, i=1, \ldots, M)$ to construct a linear system with respect to $c_{k, r}$ :

$$
g\left(x_{j}, t_{i}\right)=\sum_{k=1}^{N} \sum_{r=1}^{M} c_{k, r} F_{k, r}\left(x_{j}, t_{i}\right) \quad(j=1, \ldots, N, i=1, \ldots, M) .
$$

It turns out that the system has a lower block-triangular matrix with equal diagonal blocks. Each block is a square matrix of order $N$. Therefore, to solve the system using the Gaussian elimination, we need the number of operations which is proportional to $N^{3} M$. Solving the latter system, we obtain an approximate solution to the problem (3.4) in the form (3.10).

Calculating numerically the norm $\|v-g\|$ in the space $L_{2}(\partial \Omega \times(0, T))$ and comparing it with $\|g\|$, we get an accuracy of the approximate solution. We can also compare the values of $v(x, t)$ in (3.10) and $g(x, t)$ in (3.4) at different points of the lateral boundary $\partial \Omega \times \mathbb{R}_{+}$and take into account the maximum principle.

Similarly, replacing the exterior boundary layer $\Omega_{+}^{\mathrm{bl}}$ by the interior boundary layer $\Omega_{-}^{\mathrm{bl}}$ and considering the corresponding indicators $f_{k, r}$, we get a solution to the exterior Cauchy-Dirichlet problem in the form (3.10). 
Numerical examples. We will consider the problem (3.4) in the cylinder $\Omega_{+} \times[0, T]$, where $T=2.5, \Omega_{+}:=\left\{x=\left(x_{1}, x_{2}, x_{3}\right) \in \mathbb{R}^{3}: r:=\sqrt{x_{1}^{2}+x_{2}^{2}+x_{3}^{2}}<1\right\}$ is the unit ball, and $g(x, t):=t$. Let us construct numerical approximations of these solutions using the previous algorithm. The algorithm was implemented in C. We consider three Cartesian cubic grids with step sizes $h=1,0.5,025$. Using these grids, we produce exterior boundary layers for the unit ball $\Omega$ which consists, respectively, of either 128, or 224, or 536 cells. We choose the same number of either 128 , or 224 , or 536 boundary points belonging to the intervals connecting the centers of the boundary layer cells with the origin. To construct the boundary layer of the cylinder $\Omega_{+} \times[0, T]$, we put $M=10$. To calculate the coefficients $F_{k}\left(x_{j}\right)$ of the linear system (3.11), we evaluate the integrals (3.6) using the following known procedure of the ten-point Gaussian integration for an integral over the interval $[a, b]$ (see, e.g., [7, Section 4.5]). Solving the linear system (3.11) using the standard $L U$ decomposition for equal diagonal blocks (see, e.g., [7, Section 2.3]), we get the coefficients $c_{k, r}$. Then by (3.10), we can obtain values of the approximate solution $v(x, t)$ in different points. Integrating numerically, we get the relative error

$$
\frac{\|v-g\|_{L_{2}(\partial \Omega \times(0, T))}}{\|g\|_{L_{2}(\partial \Omega \times(0, T))}} \approx \begin{cases}0.358 & \text { for } h=1, \\ 0.031 & \text { for } h=0.5, \\ 0.0031 & \text { for } h=0.25\end{cases}
$$

Thus, the presented numerical method gives a rather accurate approximation. The results obtained so far are quite encouraging but we have considered only relatively simple test cases. We plan to consider more sophisticated cases in the near future.

\section{Acknowledgment}

The authors gratefully acknowledge the many helpful suggestions of the referee.

\section{References}

[1] M. S. Agranovič and M. I. Višik, Elliptic problems with a parameter and parabolic problems of general type, Uspekhi Matematicheskikh Nauk 19 (1964), no. 3 (117), 53-161, Russian Mathematical Surveys 19, 53-159.

[2] S. D. Eidelman and N. V. Zhitarashu, Parabolic Boundary Value Problems, Operator Theory: Advances and Applications, vol. 101, Birkhäuser, Basel, 1998.

[3] G. Grubb, Parameter-elliptic and parabolic pseudodifferential boundary problems in global $L_{p}$ Sobolev spaces, Mathematische Zeitschrift 218 (1995), no. 1, 43-90.

[4] A. Kozhevnikov, On explicit solvability of an elliptic boundary value problem and its application, Applicable Analysis 84 (2005), no. 8, 789-805.

[5] O. A. Ladyzhenskaya, V. A. Solonnikov, and N. N. Uralzeva, Linear and Quasi-Linear Equations of Parabolic Type, American Mathematical Society, Rhode Island, 1968.

[6] J.-L. Lions and E. Magenes, Non-Homogeneous Boundary Value Problems and Applications. Vol. II, Die Grundlehren der mathematischen Wissenschaften, vol. 182, Springer, New York, 1972.

[7] W. H. Press, B. P. Flannery, S. A. Teukolsky, and W. T. Vetterling, Numerical Recipes in C. The Art of Scientific Computing, Cambridge University Press, Cambridge, 1988. 


\section{On explicit and numerical solvability}

[8] V. S. Ryaben'kii, Method of Difference Potentials and Its Applications, Springer Series in Computational Mathematics, vol. 30, Springer, Berlin, 2002.

[9] L. N. Slobodeckiǔ, Generalized Sobolev spaces and their application to boundary problems for partial differential equations, Leningradskii Gosudarstvennyi Pedagogičeskii Institute Učenye Zapiski 197 (1958), 54-112.

[10] L. R. Volevich and A. R. Shirikyan, Stable and unstable manifolds for nonlinear elliptic equations with a parameter, Transactions of the Moscow Mathematical Society 2000 (2000), 97-138.

Alexander Kozhevnikov: Department of Mathematics, University of Haifa, Haifa 31905, Israel E-mail address: kogevn@math.haifa.ac.il

Olga Lepsky: Department of Mathematics, Natural Sciences Programs, Lesley Collage, Lesley University, 29 Everett Street, Cambridge, MA 02138, USA

E-mail address: olga@lesley.edu 